\title{
An XMM-Newton view of planetary nebulae in the Small Magellanic Cloud
}

\section{The X-ray luminous central star of SMP SMC 22}

\author{
S. Mereghetti ${ }^{1}$, N. Krachmalnicoff ${ }^{1,5}$, N. La Palombara ${ }^{1}$, A. Tiengo ${ }^{1}$, T. Rauch ${ }^{2}$, F. Haberl ${ }^{3}$, \\ M. D. Filipovic ${ }^{4}$, and R. Sturm ${ }^{3}$ \\ 1 INAF, Istituto di Astrofisica Spaziale e Fisica Cosmica Milano, via E. Bassini 15, 20133 Milano, Italy \\ e-mail: sandro@iasf-milano.inaf.it \\ 2 Institute for Astronomy and Astrophysics, Kepler Center for Astro and Particle Physics, Eberhard Karls University, Sand 1, \\ 3 Max Planck Institut für extraterrestrische Physik, Giessenbachstrasse 1, 85748 Garching bei München, Germany \\ ${ }^{4}$ University of Western Sydney, Locked Bag 1797, Penrith South DC, NSW 1797, Australia \\ 5 Dipartimento di Fisica, Università degli Studi di Milano, via G. Celoria 16, 20133 Milano, Italy
} 72076 Tübingen, Germany

Received 15 March 2010 / Accepted 4 May 2010

\section{ABSTRACT}

\begin{abstract}
During an X-ray survey of the Small Magellanic Cloud, carried out with the XMM-Newton satellite, we detected significant soft $\mathrm{X}$-ray emission from the central star of the high-excitation planetary nebula SMP SMC 22. Its very soft spectrum is reproduced well with a non-local thermodynamical equilibrium model atmosphere composed of $\mathrm{H}, \mathrm{He}, \mathrm{C}, \mathrm{N}$, and $\mathrm{O}$, with abundances equal to those inferred from studies of its nebular lines. The derived effective temperature of $1.5 \times 10^{5} \mathrm{~K}$ is in close agreement with that found from the optical/UV data. The unabsorbed flux in the $0.1-0.5 \mathrm{keV}$ range is $\sim 3 \times 10^{-11} \mathrm{erg} \mathrm{cm}^{-2} \mathrm{~s}^{-1}$, corresponding to a luminosity of $\sim 1.2 \times 10^{37} \mathrm{erg} \mathrm{s}^{-1}$ at a distance of $60 \mathrm{kpc}$. We also searched for X-ray emission from a large number of SMC planetary nebulae, confirming the previous detection of SMP SMC 25 with a luminosity of $(0.2-6) \times 10^{35} \mathrm{erg} \mathrm{s}^{-1}(0.1-1 \mathrm{keV})$. For the remaining objects that were not detected, we derived flux upper limits corresponding to luminosity values from several tens to hundreds times lower than that of SMP SMC 22. The exceptionally high X-ray luminosity of SMP SMC 22 is probably due to the high mass of its central star, which is rapidly evolving toward the white dwarf's cooling branch, and to a small amount of intrinsic absorption in the nebula itself.
\end{abstract}

Key words. planetary nebulae: individual: SMP SMC 22 - planetary nebulae: individual: SMP SMC 25 - Magellanic Clouds planetary nebulae: general

\section{Introduction}

Planetary nebulae (PNe) represent a common stage in the evolution of low and intermediate mass stars, which subsequently leads to the formation of white dwarfs. They are formed when the fast wind from the central star interacts with the matter of the denser wind previously ejected during the asymptotic giant branch (AGB) phase, and are characterized by $\mathrm{H}_{\alpha}$ line emission. Since the advent of imaging X-ray telescopes, a number of $\mathrm{PNe}$ have been detected in the soft $\mathrm{X}$-ray range (see, e.g., Chu et al. 2003), but due to their relatively low fluxes, detailed studies with the XMM-Newton and Chandra satellites have been carried out for only a few objects (Gruendl et al. 2006; Kastner 2009; Montez et al. 2009). The X-ray emission from PNe may originate in either their central star or the hot gas shocked in the interaction between the two stellar winds. Usually one of these two origins, characterized by different spectral and spatial signatures, is predominant, but there are also PNe in which evidence of both origins has been detected. There is also the possibility that some $\mathrm{X}$-ray emission observed from $\mathrm{PNe}$ is produced by coronal emission from a companion star (see, e.g., Soker \& Kastner 2002).

The nebula SMP SMC 22 (hereinafter SMP 22) is a highexcitation planetary nebula (PN) located in the Small Magellanic
Cloud (SMC) (Sanduleak et al. 1978; Aller et al. 1987) that has an extremely high X-ray luminosity (Wang 1991). Its high X-ray flux led to both its early detection in the soft X-ray range with the Einstein Observatory (Seward \& Mitchell 1981) and to its inclusion in the class of super-soft X-ray sources (SSS), a rather heterogeneous group of luminous $\left(10^{36}-10^{38} \mathrm{erg} \mathrm{s}^{-1}\right)$ sources characterized by thermal-like emission corresponding to black-body temperatures of $10^{5}-10^{6} \mathrm{~K}$ (see, e.g., Kahabka \& van den Heuvel 2006). Most SSS are binary systems containing accreting white dwarfs, but a few have been identified with the nuclei of PNe. Here we report on X-ray observations of SMP 22 obtained with the XMM-Newton satellite, as well as a systematic search for $\mathrm{X}$-ray emission from a large sample of PNe in the SMC.

\section{Observations and data analysis}

The field of SMP 22 has been observed on three separate occasions with XMM-Newton (see Table 1). The two 2009 pointings were obtained as part of our Large Program to perform a complete survey of the SMC (Haberl \& Pietsch 2008). For completeness, we also analyzed the 2007 observation available in the public archive. The data discussed here were obtained with the EPIC instrument, consisting of one pn and two MOS cameras 
Table 1. Log of the XMM-Newton observations of SMP 22.

\begin{tabular}{ccccccc}
\hline \hline ID & Date & $\begin{array}{c}\text { Duration } \\
(\mathrm{ks})\end{array}$ & $\begin{array}{c}\text { Net exposure } \\
(\mathrm{ks})\end{array}$ & $\begin{array}{c}\text { Off axis angle } \\
(\operatorname{arcmin})\end{array}$ & $\begin{array}{c}\text { Count rate } \\
\left(\mathrm{cts} \mathrm{s}^{-1}\right)\end{array}$ & Tot. counts \\
\hline 0501470101 & $2007 / 06 / 04$ & 31.8 & 7.7 & 5.58 & 0.400 & 3100 \\
0601210101 & $2009 / 05 / 14$ & 26.7 & 16.54 & 7.20 & 0.309 & 5167 \\
0601210501 & $2009 / 09 / 25$ & 48.7 & 32.3 & 14.06 & 0.153 & 5442 \\
\hline
\end{tabular}

Notes. All the quantities refer to the EPIC pn camera.

Table 2. Best-fit model parameters and $90 \%$ c.l. errors; $F_{\mathrm{X}}$ is the unabsorbed flux in the $0.1-0.5 \mathrm{keV}$ energy band.

\begin{tabular}{lcccccccc}
\hline \hline Mod. & $\chi_{\text {red }}^{2}$ /d.o.f. & $\begin{array}{c}N_{\mathrm{H}} \\
\left(10^{20} \mathrm{~cm}^{-2}\right)\end{array}$ & $\begin{array}{c}T \\
\left(10^{5} \mathrm{~K}\right)\end{array}$ & $\begin{array}{c}R \\
\left(10^{9} \mathrm{~cm}\right)\end{array}$ & $\alpha$ & $Z$ & $\begin{array}{c}F_{\mathrm{X}} \\
\left(10^{-10} \frac{\mathrm{erg}}{\mathrm{cm}^{2} \mathrm{~s}}\right)\end{array}$ & $\begin{array}{c}F_{\text {bol }} \\
\left(10^{-10} \frac{\mathrm{erg}}{\mathrm{cm}^{2} \mathrm{~s}}\right)\end{array}$ \\
\hline Blackbody & $0.98 / 124$ & $5.2_{-1.9}^{+1.3}$ & $3.13_{-0.23}^{+0.46}$ & $3.4_{-2.1}^{+3.4}$ & - & - & 0.9 & 1.9 \\
Brems. & $1.10 / 124$ & $4.1_{-1.0}^{+5.2}$ & $4.87 \pm 0.46$ & - & - & - & 0.6 & 12.3 \\
Mekal & $0.93 / 123$ & $5.5_{-1.7}^{+2.0}$ & $3.94 \pm 0.69$ & - & - & $0.02_{-0.01}^{+0.02}$ & 3.0 & 86.1 \\
Pow. law & $1.69 / 124$ & $\geq 4.89$ & - & - & $\geq 9.4$ & - & $\geq 22.6$ & - \\
\hline
\end{tabular}

(Strüder et al. 2001; Turner et al. 2001) covering a field of view of about $30^{\prime}$ diameter in the energy range $0.1-12 \mathrm{keV}$. In all the observations, they were operated in full frame mode, yielding a time resolution of $73 \mathrm{~ms}$ for the pn and $2.6 \mathrm{~s}$ for MOS 1 and MOS2. We processed the data with the standard XMM-Newton Science Analysis Software (SAS, version 8.0). We filtered out time intervals affected by high background, induced by soft protons, resulting in the net exposure times listed in Table 1.

To extract spectra and light curves, we selected only single and double events of energy between 0.1 and $12 \mathrm{keV}$. For the first and second observations, we used circular extraction regions with radii of $30^{\prime \prime}$ and $25^{\prime \prime}$, respectively. In the third observation, because of the large off-axis angle, SMP 22 appears to have an elongated shape; for this reason, we chose an elliptical region to extract the source events. In all cases, the background spectrum was obtained from events extracted in a large area with no sources in the same CCD containing SMP 22. We generated the response matrices ( $r m f$ ) and the ancillary files (arf) using the SAS tasks RMFGEN and ARFGEN. All source spectra extracted from the event lists were rebinned to ensure a minimum of 30 counts per energy bin, and fitted using the XSPEC program. A systematic error of 5\% was included in the spectral fits to account for the known uncertainties ${ }^{1}$ in the response matrices below $0.5 \mathrm{keV}$.

The light curves extracted for SMP 22 are consistent with there being a constant flux in each data set, and the source count rates in the three observations, when corrected for the different off-axis angle, do not show any evidence of long-term variability.

The two MOS cameras together provide only about $10 \%$ of the total counts detected from the source and the addition of their spectra does not significantly improve the results. Therefore, in the following we report only the results obtained with the pn. The spectra from the three observations were fitted simultaneously, using common parameters (except for the relative normalization, whose values were found to be always within $1 \%$ ).

The results obtained with a few single component models confirm that the source spectrum is very soft (see Table 2), practically no source counts being detected above $0.5 \mathrm{keV}$. A black body or a thermal plasma model (Mekal in XSPEC) provide the best fits, with temperatures of about $(3-5) \times 10^{5} \mathrm{~K}$

\footnotetext{
1 EPIC status of calibration and data analysis, http://xmm2 . esac . esa. int/docs/documents/CAL-TN-0018.pdf
}

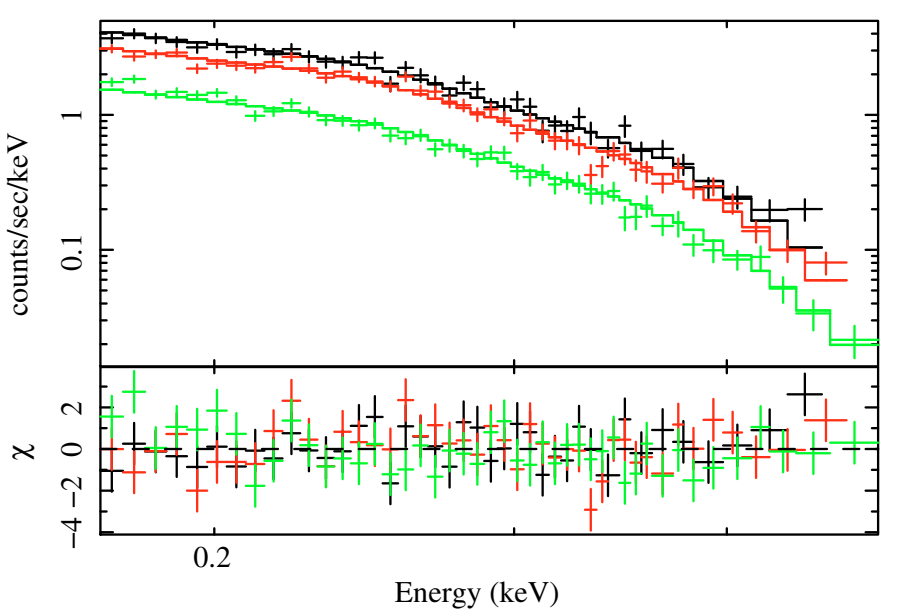

Fig. 1. Spectra of SMP 22 fitted with a black-body model. Top panel: data points and best-fit model for the three observations (in chronological order from the highest to lowest count rate). Bottom panel: residuals from the best-fit model in units of $\sigma$.

and absorption $N_{\mathrm{H}} \sim 5 \times 10^{20} \mathrm{~cm}^{-2}$. A power law, besides giving an unacceptable $\chi^{2}$, yields an unrealistic photon index. The best-fit black-body model is shown in Fig. 1, and the corresponding confidence contours of temperature and absorption in Fig. 2. At a distance of $60 \mathrm{kpc}$ (adopted hereinafter; see, e.g., Deb \& Singh 2010), the black-body emitting surface corresponds to a star radius of $0.05 R_{\odot}$ and the bolometric luminosity is $\sim 8 \times 10^{37} \mathrm{erg} \mathrm{s}^{-1}$.

We also explored fits with model atmospheres computed under non-local thermodynamic equilibrium (NLTE) conditions. A small grid of model atmospheres was calculated with $T M A P^{2}$, the Tübingen Model-Atmosphere Package (Werner et al. 2003; Rauch \& Deetjen 2003). These models, for a fixed chemical composition, have $\log g$ and $T_{\text {eff }}$ as free parameters, and were converted to the XSPEC atable format for spectral fitting. In the framework of the Virtual Observatory, the spectral energy distributions (SEDs) of these models are available via the German Astrophysical Virtual Observatory (GAVO) service TheoSSA ${ }^{3}$.

\footnotetext{
2 http://astro.uni-tuebingen.de/ rauch/TMAP/TMAP.html

3 http://vo.ari.uni-heidelberg.de/ssatr-0.01/

TrSpectra.jsp?
} 


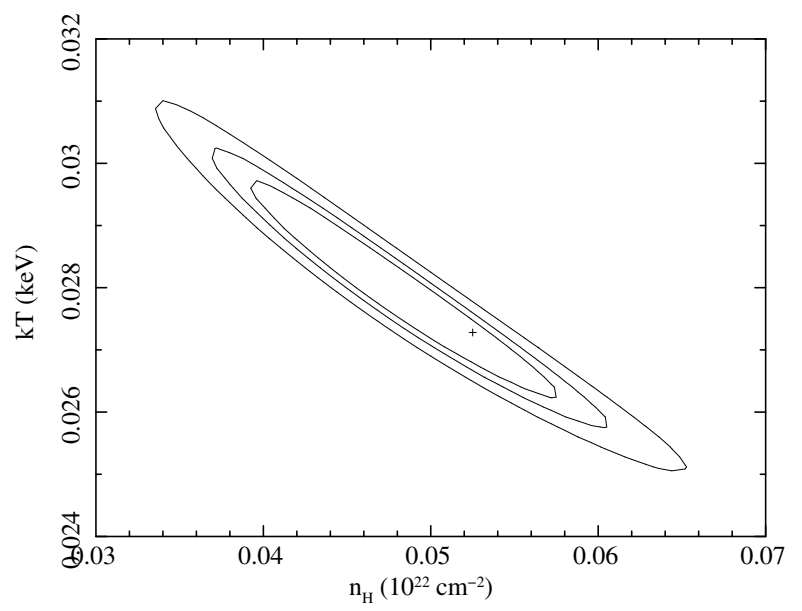

Fig. 2. Confidence contours $(68 \%, 90 \%$, and $99 \%$ c.1.) for the absorption and temperature obtained with the black-body fit to the three joint observations.

Table 3. Element abundances used in the NLTE model atmospheres.

\begin{tabular}{cc}
\hline \hline \multicolumn{2}{c}{ Mass fraction } \\
\hline $\mathrm{H}$ & $6.808 \times 10^{-1}$ \\
$\mathrm{He}$ & $3.176 \times 10^{-1}$ \\
$\mathrm{C}$ & $9.104 \times 10^{-5}$ \\
$\mathrm{~N}$ & $1.061 \times 10^{-3}$ \\
$\mathrm{O}$ & $4.205 \times 10^{-4}$ \\
\hline
\end{tabular}

Adopting a pure $\mathrm{H}$ composition, good fits could be obtained for effective temperatures of $(0.7-1.1) \times 10^{5} \mathrm{~K}$, but with unconstrained values of gravity in the wide range $5<\log g<9$ covered by the models. Equally good fits were provided by a $\mathrm{He}$ atmosphere with $T_{\text {eff }}=(1-1.2) \times 10^{5} \mathrm{~K}$, and in this case the surface gravity was constrained to be $\log g \sim 5.5 \pm 0.5$. The best-fit effective temperatures of the $\mathrm{H}$ and He NLTE model fits imply that the source emitting radii must be $\sim 1-10 R_{\odot}$, to produce the observed source luminosity. These radii, coupled with their corresponding $\log g$ values, imply unrealistically high values of mass for the star $\left(>100 M_{\odot}\right)$. We thus conclude that, although the $\mathrm{H}$ and He NLTE model atmospheres provide formally good fits to the EPIC data, they are physically unacceptable.

We finally considered a NLTE model atmosphere that includes other elements. Since the limited spectral resolution of our data does not allow a detailed determination of the elemental composition and abundances, we restricted our analysis to a single model, based on the results of optical/UV spectroscopy of the SMP 22 nebular emission. We therefore adopted a model composed of $\mathrm{H}, \mathrm{He}, \mathrm{C}, \mathrm{N}$, and $\mathrm{O}$ with abundances fixed to the values determined by Leisy \& Dennefeld (1996), and reported in Table 3. The results obtained with this model are summarized in Table 4, where, for three fixed values of $\log g$, we report the interstellar absorption, effective temperature, and radius of the emission surface derived from the fit (Cols. 3-5), and the star's mass implied by the $\log g$ and $R$ values (Col. 6).

In all cases, an effective temperature of $T_{\text {eff }} \sim 1.5 \times 10^{5} \mathrm{~K}$ is obtained. Although formally the best-fit model is found for $\log g=7$, the $\log g=6$ case (Fig. 3) is preferable because it is only marginally worse but provides more plausible values of mass $\left(\sim 1 M_{\odot}\right)$ and radius $\left(\sim 0.2 R_{\odot}\right)$. We checked that the flux in the visible band predicted by this model is lower $(\sim 30 \%)$ than the current upper limit obtained with the Hubble Space Telescope for the PN central star (Villaver et al. 2004).

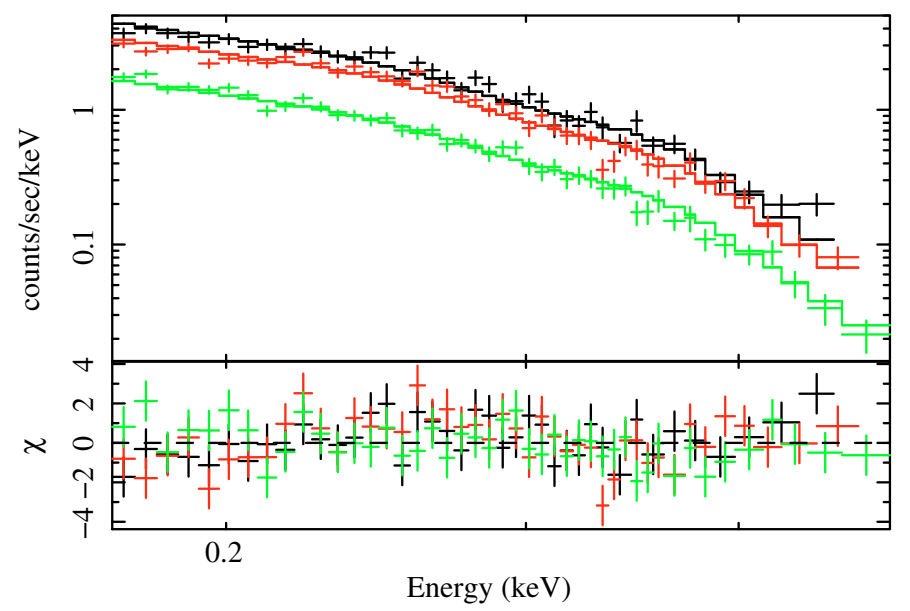

Fig. 3. Spectra of SMP 22 fitted with a NLTE model atmosphere with $\log g=6$ (see Table 4). Top panel: data points and model. Bottom panel: residuals from the model in units of $\sigma$.

\subsection{Search for $X$-ray emission from other SMC planetary nebulae}

Based on several compilations (see references in Table 5), we selected all the PNe in the SMC that had been observed by $X M M$-Newton in our survey or during other observations in the public archive. Using the data from the EPIC pn camera, we searched for X-ray emission from these objects in the $0.1-1 \mathrm{keV}$ energy band by applying the SAS maximum likelihood source detection task EMLDETECT with the threshold parameter mlmin set to the value of 6 . For PNe observed in more than one pointing, typically with different off-axis angles and net exposure times, we used the data of the highest sensitivity at the source position. With the exception of SMP SMC 25 (see below), no source in our sample was significantly detected. We report in Table 5 the upper limits on their $0.1-1 \mathrm{keV}$ count rates, which take into account the vignetting correction required because of the different off-axis angles. The conversion of these values to physical units was based on assumptions about the source spectra. Conversion factors from count rate to flux, for different values of temperature and hydrogen column density, are plotted in Fig. 4 for the case of a thin optical filter (that was used in most observations). As representative examples, we report in Table 5 the luminosity upper limits of a black-body model with $k T=$ $20 \mathrm{eV}$ and a bremsstrahlung plasma model with $k T=100 \mathrm{eV}$ (in both cases $N_{\mathrm{H}}=5 \times 10^{20} \mathrm{~cm}^{-2}$ ). For comparison, the respective values for SMP 22 would be $L_{\mathrm{BB}}=1.9 \times 10^{38} \mathrm{erg} \mathrm{s}^{-1}$ and $L_{\mathrm{TB}}=2.2 \times 10^{36} \mathrm{erg} \mathrm{s}^{-1}$.

The detection of SMP SMC 25 with XMM-Newton confirms the association of the low-luminosity SSS source RX J0059.6-7138, discovered with ROSAT (Kahabka et al. 1999), with this planetary nebula, also known as LIN357 (Meyssonnier \& Azzopardi 1993). The coordinates derived with EPIC $\left(\mathrm{RA}=0^{\mathrm{h}} 59^{\mathrm{m}} 40.3^{\mathrm{s}}\right.$, Dec $=-71^{\circ} 38^{\prime} 17^{\prime \prime}$, with a $1 \sigma$ uncertainty of 2.5") are consistent with the optical position of SMP SMC 25. For comparison, the $90 \%$ c.l. error radius obtained for RX J0059.6-7138 with ROSAT was 14.5" (Haberl et al. 2000). The EPIC and ROSAT count rates, considering the uncertainties caused by the small number statistics and poorly constrained spectral properties, are consistent with a constant source flux. 
A\&A 519, A42 (2010)

Table 4. Fit parameters for a $\mathrm{H} / \mathrm{He} / \mathrm{C} / \mathrm{N} / \mathrm{O}$ NLTE atmosphere; $F_{\mathrm{X}}$ is the unabsorbed source flux in the $0.1-0.5 \mathrm{keV}$ energy band.

\begin{tabular}{cccccccc}
\hline \hline $\begin{array}{c}\log g \\
\left(\mathrm{~cm} \mathrm{~s}^{-2}\right)\end{array}$ & $\chi_{\text {red }}^{2} /$ d.o.f. & $\begin{array}{c}N_{\mathrm{H}} \\
\left(10^{20} \mathrm{~cm}^{-2}\right)\end{array}$ & $\begin{array}{c}T \\
\left(10^{5} \mathrm{~K}\right)\end{array}$ & $\begin{array}{c}R \\
\left(10^{10} \mathrm{~cm}\right)\end{array}$ & $\begin{array}{c}M \\
\left(10^{33} \mathrm{~g}\right)\end{array}$ & $\begin{array}{c}F_{\mathrm{X}} \\
\left(10^{-11} \frac{\mathrm{erg}}{\mathrm{cm}^{2} \mathrm{~s}}\right)\end{array}$ & $\begin{array}{c}F_{\text {bol }} \\
\left(10^{-10} \frac{\text { erg }}{\mathrm{cm}^{2} \mathrm{~s}}\right)\end{array}$ \\
\hline 6.0 & $1.17 / 124$ & $2.8 \pm 0.4$ & $1.54 \pm 0.01$ & $1.3 \pm 0.1$ & $2.4 \pm 0.2$ & 2.96 & 1.51 \\
6.5 & $1.11 / 124$ & $3.2_{-0.5}^{+0.3}$ & $1.54_{-0.02}^{+0.01}$ & $1.5 \pm 0.1$ & $10.1 \pm 0.6$ & 3.5 & 2.0 \\
7.0 & $1.09 / 124$ & $3.0 \pm 0.5$ & $1.54_{-0.02}^{+0.01}$ & $1.3 \pm 0.1$ & $25.1_{-1.3}^{+2.2}$ & 2.73 & 1.56 \\
\hline
\end{tabular}

Table 5. $3 \sigma$ upper limits for planetary nebulae in the SMC observed with XMM-Newton (Except for SMP SMC 25 for which we report the detected count rate).

\begin{tabular}{|c|c|c|c|c|}
\hline Name $^{(1)}$ & $\begin{array}{c}\text { Rate }^{(2)} \\
\left(10^{-3} \text { cts s}^{-1}\right)\end{array}$ & $\begin{array}{c}L_{\mathrm{BB}}^{(3)} \\
\left(10^{34} \mathrm{erg} \mathrm{s}^{-1}\right)\end{array}$ & $\begin{array}{c}L_{\mathrm{TB}}^{(4)} \\
\left(10^{34} \mathrm{erg} \mathrm{s}^{-1}\right)\end{array}$ & Observation date \\
\hline SMP SMC 5 & 3.7 & 67.5 & 2.5 & $2006 / 03 / 27$ \\
\hline SMP SMC 7 & 1.1 & 20.5 & 0.8 & $2009 / 10 / 20$ \\
\hline SMP SMC 8 & 2.2 & 40.6 & 1.5 & 2009/11/09 \\
\hline SMP SMC 9 & 1.5 & 27.5 & 1.0 & 2009/10/03 \\
\hline SMP SMC 10 & 2.9 & 53.5 & 2.0 & 2009/09/27 \\
\hline SMP SMC $12^{*}$ & 3.2 & 93.9 & 2.7 & $2007 / 10 / 28$ \\
\hline SMP SMC $13^{*}$ & 1.7 & 50.6 & 1.5 & 2007/10/28 \\
\hline SMP SMC $14^{*}$ & 2.6 & 75.0 & 2.2 & $2007 / 10 / 28$ \\
\hline SMP SMC 16 & 2.5 & 45.2 & 1.7 & $2007 / 06 / 23$ \\
\hline SMP SMC 18 & 2.3 & 43.2 & 1.6 & 2007/04/11 \\
\hline SMP SMC 19 & 1.6 & 29.5 & 1.1 & 2006/11/01 \\
\hline SMP SMC 21 & 1.7 & 31.4 & 1.2 & 2009/10/09 \\
\hline SMP SMC 23 & 1.9 & 35.0 & 1.3 & 2009/10/11 \\
\hline SMP SMC 25 & $3.2 \pm 0.9$ & 58.5 & 2.2 & 2007/06/04 \\
\hline SMP SMC 27 & 1.2 & 22.9 & 0.9 & $2009 / 06 / 29$ \\
\hline $\mathrm{J} 2$ & 1.8 & 33.9 & 1.3 & 2009/10/03 \\
\hline $\mathrm{J} 4^{*}$ & 3.5 & 102.3 & 3.0 & $2007 / 10 / 28$ \\
\hline J5 & 2.8 & 51.6 & 1.9 & 2009/10/03 \\
\hline J9 & 3.0 & 56.2 & 2.1 & 2007/04/11 \\
\hline $\mathrm{J} 10$ & 1.9 & 34.7 & 1.3 & $2009 / 09 / 27$ \\
\hline $\mathrm{J} 12$ & 1.8 & 33.7 & 1.3 & $2009 / 09 / 27$ \\
\hline $\mathrm{J} 13$ & 7.0 & 129.1 & 4.8 & $2007 / 06 / 23$ \\
\hline $\mathrm{J} 15$ & 2.3 & 42.4 & 1.6 & $2007 / 06 / 23$ \\
\hline J16 & 2.5 & 46.1 & 1.7 & $2007 / 06 / 23$ \\
\hline $\mathrm{J} 17$ & 2.1 & 39.3 & 1.5 & 2007/04/11 \\
\hline $\mathrm{J} 18$ & 2.0 & 36.7 & 1.4 & 2006/11/01 \\
\hline $\mathrm{J} 21$ & 1.6 & 28.6 & 1.1 & 2009/11/04 \\
\hline $\mathrm{J} 22$ & 3.2 & 58.5 & 2.2 & $2007 / 06 / 23$ \\
\hline $\mathrm{J} 23$ & 12.7 & 234.2 & 8.7 & 2009/10/11 \\
\hline $\mathrm{J} 24$ & 4.4 & 80.8 & 3.0 & 2009/10/11 \\
\hline $\mathrm{J} 25$ & 1.7 & 30.8 & 1.1 & 2009/10/11 \\
\hline $\mathrm{J} 27$ & 2.4 & 43.5 & 1.6 & 2009/10/11 \\
\hline MG8 & 1.6 & 29.1 & 1.1 & 2009/09/27 \\
\hline MG9 & 3.6 & 66.9 & 2.5 & 2007/06/06 \\
\hline MG10 & 3.2 & 59.4 & 2.2 & $2006 / 10 / 03$ \\
\hline MG11 & 1.6 & 29.1 & 1.1 & 2009/11/30 \\
\hline MA14 & 2.1 & 38.0 & 1.4 & $2009 / 10 / 20$ \\
\hline MA44 & 1.8 & 33.0 & 1.2 & 2009/10/18 \\
\hline MA406* & 3.0 & 86.9 & 2.5 & $2007 / 10 / 28$ \\
\hline MA891 & 4.0 & 74.5 & 2.8 & $2002 / 03 / 30$ \\
\hline MA1357 & 4.2 & 76.9 & 2.9 & 2009/10/16 \\
\hline MA1714 & 1.6 & 29.0 & 1.1 & 2009/11/16 \\
\hline MA1762 & 2.6 & 47.4 & 1.8 & 2009/11/19 \\
\hline M7 & 1.9 & 36.0 & 1.3 & $2009 / 06 / 29$ \\
\hline JD29 & 1.7 & 31.5 & 1.2 & 2009/10/18 \\
\hline JD51 & 2.8 & 51.8 & 1.9 & 2009/10/09 \\
\hline JD53 & 3.5 & 64.2 & 2.4 & 2007/06/06 \\
\hline JD57 & 1.3 & 24.2 & 0.9 & 2009/09/25 \\
\hline
\end{tabular}

Notes. The sources marked by a star were observed with the medium optical filter, all the others with the thin one. (1) The names indicate the following references: SMP = Sanduleak et al. (1978), J = Jacoby (1980), MG = Morgan \& Good (1985), MA = Meyssonnier \& Azzopardi (1993), $\mathrm{M}=$ Morgan (1995), JD = Jacoby \& De Marco (2002); (2) 0.1-1 keV count rate in the EPIC pn camera; (3) unabsorbed 0.1-1 keV luminosity for a black-body spectrum with $k T=20 \mathrm{eV}, N_{\mathrm{H}}=5 \times 10^{20} \mathrm{~cm}^{-2}$, and $d=60 \mathrm{kpc}$; (4) as (3) but for a thermal bremsstrahlung with $k T=100 \mathrm{eV}$. 

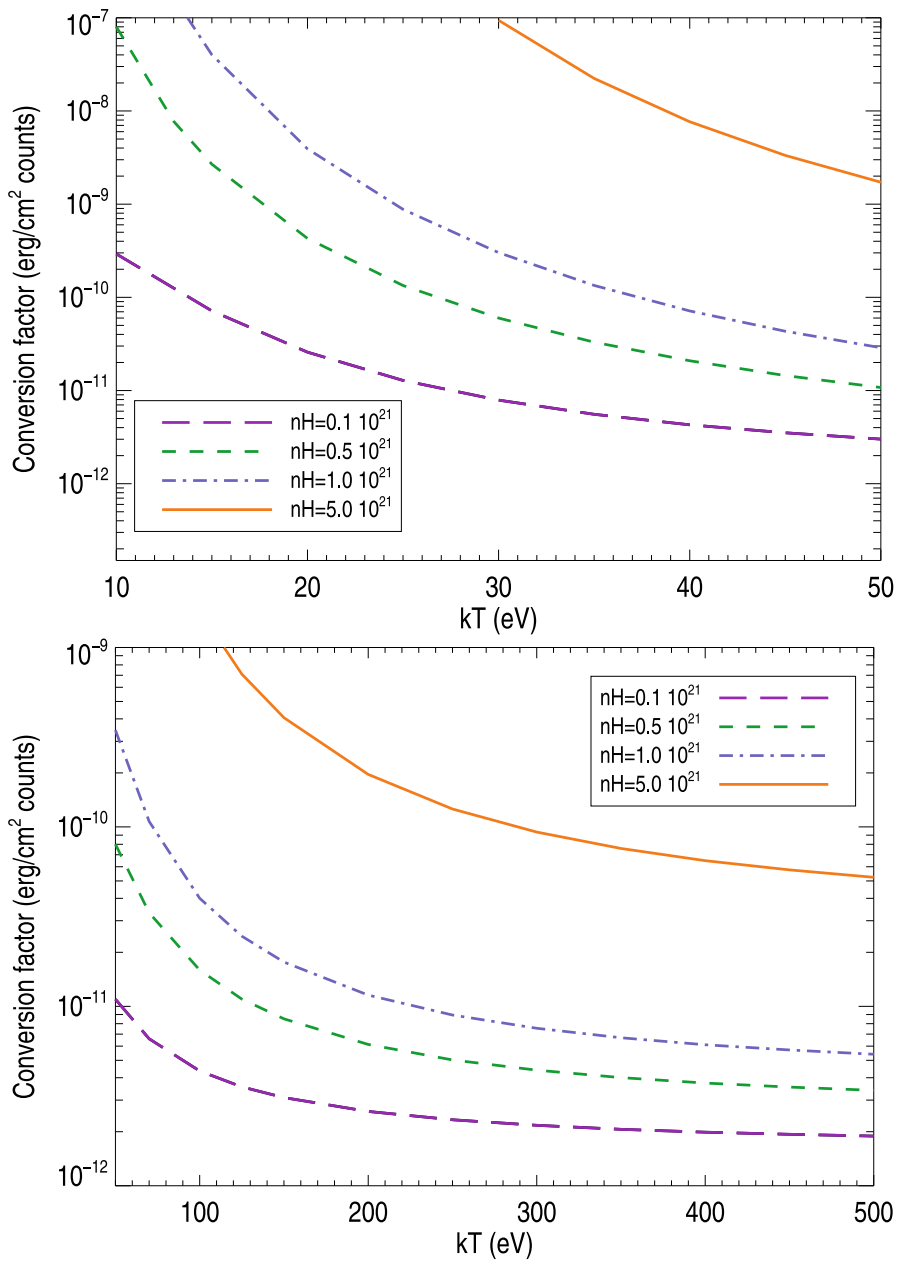

Fig. 4. Conversion factors from PN count rate to unabsorbed flux in the 0.1-1 keV energy band. Top panel: black-body model; bottom panel: bremsstrahlung model. Both plots refer to observations carried out with the thin optical filter.

\section{Discussion}

Owing to its location in the SMC, the PN SMP 22 has too small angular dimensions to be spatially resolved with EPIC, being $0.71^{\prime \prime} \times 0.54^{\prime \prime}$ (Stanghellini et al. 2003; Vassiliadis et al. 1998). However, its very soft spectrum and high luminosity derived by our spectral analysis imply that its X-ray emission originates in the central star, without any significant contribution from the diffuse gas. The black-body fit implies a bolometric luminosity $L_{\text {bol }} \sim 8 \times 10^{37} \mathrm{erg} \mathrm{s}^{-1}$, while the more realistic atmosphere model with the nebular abundances yields $L_{\mathrm{bol}}=6 \times 10^{37} \mathrm{erg} \mathrm{s}^{-1}$. This value is orders of magnitude higher than the luminosity that can be produced in the surrounding shock-heated gas. The same conclusion was reached by Wang (1991), who, because of a lack of adequate X-ray spectral information, assumed a black body with $T=3 \times 10^{5} \mathrm{~K}$.

Previous spectral analyses of SMP 22 were carried out using data from both the Einstein Observatory by Brown et al. (1994) and ROSAT by Kahabka et al. (1994). A black-body model was used in both cases, yielding best-fit model parameters consistent with, but far less tightly constrained, than those derived here in our analysis. Comparing these data, spanning almost 30 years, we find no evidence of long-term variability in the source luminosity or spectrum. Heise et al. (1994) fitted the ROSAT spectrum of SMP 22 with models of H-rich and

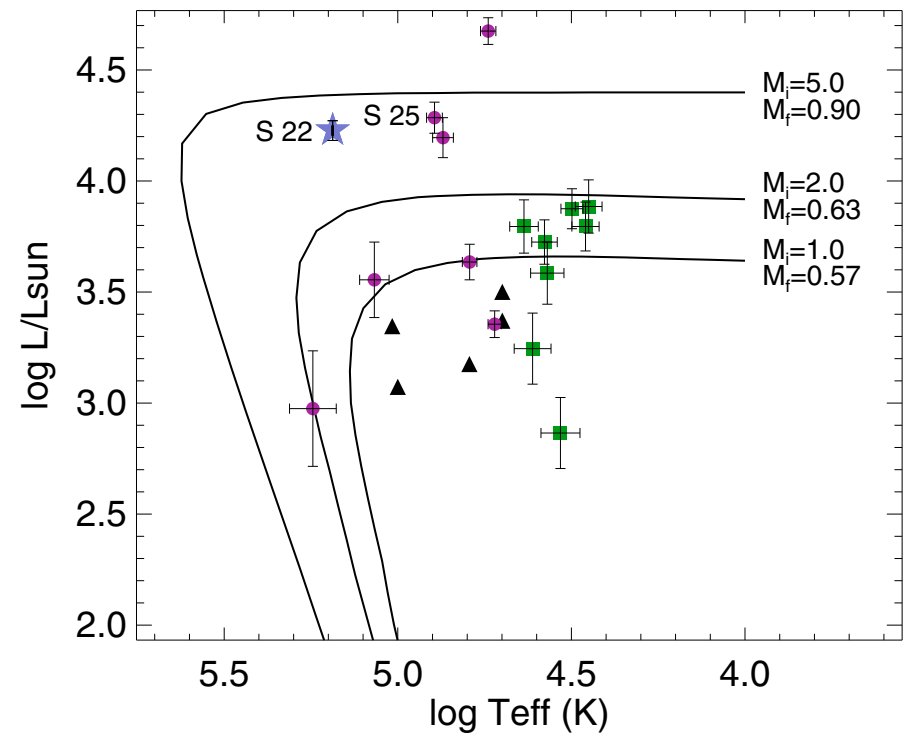

Fig. 5. H-R diagram for central stars of PNe in the SMC. Black triangles are from Aller et al. (1987), purple circles and green squares from Villaver et al. (2004) (Zanstra He and H temperature respectively). The blue star represents the nucleus of SMP 22. Evolutionary tracks for SMC metallicity are from Vassiliadis \& Wood (1994).

He-rich white dwarf atmospheres computed with the LTE assumption. They found that these models, appropriate for $\mathrm{H}-$ or He-burning accreting white dwarfs, yield a bolometric luminosity of $\sim 2 \times 10^{37} \mathrm{erg} \mathrm{s}^{-1}$, more than one order of magnitude lower than the super-Eddington value implied by the black-body fit to the same data. However, the lack of variability and the upper limit $(V>20.7)$ to the optical counterpart (Villaver et al. 2004), do not favor a binary nature, in contrast to the case for other SSS. The good fit provided by our NLTE model instead supports the interpretation of SMP 22 as a single, very hot star on its way to becoming a relatively massive $\left(\sim 1 M_{\odot}\right)$ white dwarf. Additional evidence of a high mass star is the high N/O ratio (see Table 3 ), which is consistent with a type I PN, and indicative of a massive progenitor (see, e.g., Stanghellini 2007).

It is interesting to compare the temperature of the central star derived from our X-ray spectral analysis $\left(T \sim 1.5 \times 10^{5} \mathrm{~K}\right)$ with the values obtained from the modeling of nebular emission lines observed at optical/UV wavelengths. Based on the Zanstra method, which assumes a black-body spectrum for the ionizing radiation from the central star (Zanstra 1927), Villaver et al. (2004) derived $T_{\mathrm{HeII}}=(1.222 \pm 0.145) \times 10^{5} \mathrm{~K}$ and $T_{\mathrm{H}}=(0.77 \pm 0.18) \times 10^{5} \mathrm{~K}$, using the He II $4686 \AA$ and $\mathrm{H}_{\beta}$ lines, respectively. A temperature $T=1.15 \times 10^{5} \mathrm{~K}$ was instead obtained from IUE spectroscopy, by adopting NLTE model atmospheres (Aller et al. 1987). These values are in good agreement with our results, considering the unavoidable uncertainties intrinsic in all these model-dependent estimates.

The X-ray detection of SMP 22 is unsurprising, because all the studies based on optical/UV data indicate that this PN hosts one of the hottest central stars. On the other hand, the same studies of the SMC also detect other PNe of similarly high temperature, as illustrated by the H-R diagram plotted in Fig. 5. Based on this plot, we would expect to find other objects with a soft X-ray emission comparable to, or slightly lower than, that of SMP 22. Instead, our results (Table 5) indicate that most of these PNe are more than two orders of magnitude less luminous in the X-ray band than SMP 22. This result also applies to SMP SMC 25, which has a Zanstra temperature of about a factor of two lower 
than that of SMP 22 (Villaver et al. 2004), accounting for the large difference in the soft X-ray flux between these two PNe. Thus, the X-ray faintness of SMC PNe with hot central stars is probably caused by the combination of absorption in the nebula and the strong dependence of the flux in the EPIC soft X-ray band on the temperature (see Fig. 4). The small value of absorption derived from our fits of SMP 22 is consistent with the Galactic value in the SMC direction, and indicative of only little or no intrinsic absorption in the nebula itself. The high temperature and luminosity of SMP 22 are also the likely explanation of the absence of dust-related features in the Spitzer infrared data (Bernard-Salas et al. 2009). As demonstrated by its IR Spitzer spectra, SMP 22 is one of the most energetic PNe with high excitation lines ([O IV] and [Ne V]). However, it is not a unique object, as several of the SMC PNe in the study of Bernard-Salas et al. (2009) show these lines. This also supports our argument that the absorption may play a major role in hiding other massive PNe across the whole SMC. Filipović et al. (2009) and Payne et al. (2008) detected four radio-continuum PNe in the SMC, which also appear to have very large and massive central stars. They even tentatively called them "super PNe" because of their extraordinary nature. We searched all available radio-continuum images and catalogues at various radio frequencies and found no emission from SMP 22 and SMP SMC 25 down to a $3 \sigma$ limit of 0.3 mJy (Filipović et al. 1997, 1998, 2002).

PNe in our Galaxy have been observed to have X-ray luminosities reaching at most $\sim 10^{32} \mathrm{erg} \mathrm{s}^{-1}$ (Kastner 2009), but the detection of super soft sources such as SMP 22 and SMP SMC 25 in the Galactic plane direction is hampered by interstellar absorption. Unsurprisingly, the PN most closely resembling SMP 22 in its X-ray properties has been found in the Large Magellanic Cloud: XMM-Newton observations reported by Kahabka et al. (2008) showed that SMP LMC 29 has a soft $\mathrm{X}$-ray spectrum that could be well described by a black-body with $T$ in the range $(3-6) \times 10^{5} \mathrm{~K}$ and a bolometric luminosity of $(0.1-30) \times 10^{36} \mathrm{erg} \mathrm{s}^{-1}$.

\section{Conclusions}

The first X-ray observations of the SMC planetary nebula SMP 22 obtained with a modern high-throughput satellite have allowed us to study its X-ray emission with unprecedented statistics. We have found no evidence of a binary nature, such as longor short-term variability, as seen in other SSS. It is remarkable that, despite different spectral models being able to fit the data, a self-consistent picture in terms of temperature, mass, and radius of the central star can be obtained with a NLTE model atmosphere with the same elemental abundances as seen in the nebula. The inferred mass for the central star, of the order of $1 M_{\odot}$, implies that SMP 22 is the descendent of a relatively massive progenitor (see Fig. 5). This may explain its exceptional luminosity, as well as the apparent rarity of these objects, which evolve very quickly toward the cooling white dwarf sequence.
Acknowledgements. This work is based on observations obtained with $X M M-N e w t o n$, an ESA science mission with instruments and contributions directly funded by ESA Member States and NASA. The XMM-Newton data analysis is supported by the Italian Space Agency (ASI). T.R. is supported by the German Aerospace Center (DLR) under grant 05 OR 0806.

\section{References}

Aller, L. H., Keyes, C. D., Maran, S. P., et al. 1987, ApJ, 320, 159

Bernard-Salas, J., Peeters, E., Sloan, G. C., et al. 2009, ApJ, 699, 1541

Brown, T., Cordova, F., Ciardullo, R., Thompson, R., \& Bond, H. 1994, ApJ, 422, 118

Chu, Y., Guerrero, M. A., \& Gruendl, R. A. 2003, in Planetary Nebulae: Their Evolution and Role in the Universe, ed. S. Kwok, M. Dopita, \& R. Sutherland, IAU Symp., 209, 415

Deb, S., \& Singh, H. P. 2010, MNRAS, 402, 691

Filipović, M. D., Jones, P. A., White, G. L., et al. 1997, A\&AS, 121, 321

Filipović, M. D., Haynes, R. F., White, G. L., \& Jones, P. A. 1998, A\&AS, 130, 421

Filipović, M. D., Bohlsen, T., Reid, W., et al. 2002, MNRAS, 335, 1085

Filipović, M. D., Cohen, M., Reid, W. A., et al. 2009, MNRAS, 399, 769

Gruendl, R. A., Guerrero, M. A., Chu, Y., \& Williams, R. M. 2006, ApJ, 653, 339

Haberl, F., \& Pietsch, W. 2008, in X-rays From Nearby Galaxies, ed. S. Carpano, M. Ehle, \& W. Pietsch, 32

Haberl, F., Filipović, M. D., Pietsch, W., \& Kahabka, P. 2000, A\&AS, 142, 41

Heise, J., van Teeseling, A., \& Kahabka, P. 1994, A\&A, 288, L45

Jacoby, G. H. 1980, ApJS, 42, 1

Jacoby, G. H., \& De Marco, O. 2002, AJ, 123, 269

Kahabka, P., \& van den Heuvel, E. P. J. 2006, Super-soft sources, ed. W. H. G. Lewin, \& M. van der Klis, 461

Kahabka, P., Pietsch, W., \& Hasinger, G. 1994, A\&A, 288, 538

Kahabka, P., Pietsch, W., Filipović , M. D., \& Haberl, F. 1999, A\&AS, 136, 81

Kahabka, P., Haberl, F., Pakull, M., et al. 2008, A\&A, 482, 237

Kastner, J. H. 2009, in Conf. Proc. "Asymmetrical planetary nebulae IV", eds.

R.L.M. Corradi et al., IAC Electronic Publication [arXiv:0709.4136]

Leisy, P., \& Dennefeld, M. 1996, A\&AS, 116, 95

Meyssonnier, N., \& Azzopardi, M. 1993, A\&AS, 102, 451

Montez, R., Kastner, J. H., Balick, B., \& Frank, A. 2009, ApJ, 694, 1481

Morgan, D. H. 1995, A\&AS, 112, 445

Morgan, D. H., \& Good, A. R. 1985, MNRAS, 213, 491

Payne, J. L., Filipovic, M. D., Millar, W. C., et al. 2008, Serb. Astron. J., 177, 53

Rauch, T., \& Deetjen, J. L. 2003, in Stellar Atmosphere Modeling, ed. I. Hubeny, D. Mihalas, \& K. Werner, ASP Conf. Ser., 288, 103

Sanduleak, N., MacConnell, D. J., \& Philip, A. G. D. 1978, PASP, 90, 621

Seward, F. D., \& Mitchell, M. 1981, ApJ, 243, 736

Soker, N., \& Kastner, J. H. 2002, ApJ, 570, 245

Stanghellini, L. 2007, in Why Galaxies Care About AGB Stars: Their Importance as Actors and Probes, ed. F. Kerschbaum, C. Charbonnel, \& R. F. Wing, ASP Conf. Ser., 378, 456

Stanghellini, L., Shaw, R. A., Balick, B., et al. 2003, ApJ, 596, 997

Strüder, L., Briel, U., Dennerl, K., et al. 2001, A\&A, 365, L18

Turner, M. J. L., Abbey, A., Arnaud, M., et al. 2001, A\&A, 365, L27

Vassiliadis, E., \& Wood, P. R. 1994, ApJS, 92, 125

Vassiliadis, E., Dopita, M. A., Meatheringham, S. J., et al. 1998, ApJ, 503, 253

Villaver, E., Stanghellini, L., \& Shaw, R. A. 2004, ApJ, 614, 716

Wang, Q. 1991, MNRAS, 252, 47P

Werner, K., Deetjen, J. L., Dreizler, S., et al. 2003, in Stellar Atmosphere Modeling, ed. I. Hubeny, D. Mihalas, \& K. Werner, ASP Conf. Ser., 288, 31

Zanstra, H. 1927, ApJ, 65, 50 